\title{
Epidemiology of Neisseria gonorrhoeae Gyrase A Genotype, Los Angeles, California, USA
}

\author{
Ashima A. Bhatti, Lao-Tzu Allan-Blitz, \\ Mariana Castrejon, Romney M. Humphries, \\ Peera Hemarajata, Jeffrey D. Klausner
}

We investigated the epidemiology of the mutant gyrase A gene, a reliable predictor of ciprofloxacin resistance, in Neisseria gonorrhoeae infections at UCLA Health in Los Angeles, California, USA, during November 1, 2015-August 31, 2016. Among 110 patients with $N$. gonorrhoeae infections, 48 (44\%) had the mutant gyrase A gene.

$\mathrm{I}$ n 2013, the Centers for Disease Control and Prevention (Atlanta, GA, USA) declared that multidrug-resistant Neisseria gonorrhoeae infections were 1 of the top 3 urgent antimicrobial drug resistance threats (1). To combat this growing threat, we developed and implemented a real-time reverse transcription PCR at the University of California, Los Angeles (Los Angeles, CA, USA), to detect the codon 91 mutation in the gyrase A ( gyrA) gene in $N$. gonorrhoeae remnant clinical specimens (2). Mutations in the gyrA gene of $N$. gonorrhoeae, specifically at codon 91, have been demonstrated to reliably predict resistance to ciprofloxacin (3).

Current clinical practice uses mostly nucleic acid amplification tests to detect $N$. gonorrhoeae and not culturebased methods (4). Although those tests yield better clinical outcomes, they do not provide useful antimicrobial drug susceptibility data. Therefore, factors associated with antimicrobial drug resistance in the general community are not routinely available $(4,5)$.

\section{The Study}

We characterized the epidemiology of fluoroquinolone resistance at UCLA Health (Los Angeles, CA, USA) by using a rapid molecular gyrA gene assay that predicts ciprofloxacin susceptibility $(3,6)$. UCLA Health is a large healthcare system in Los Angeles County composed of 2 hospitals, 2 emergency departments, and $>150$ primary care clinics serving $\approx 500,000$ patient-visits each year. We reviewed electronic patient medical records for November 1, 2015-August 31, 2016, for all cases of $N$. gonorrhoeae

Author affiliations: University of California, Los Angeles, California, USA (A.A. Bhatti, L.-T. Allan-Blitz, M. Castrejon, R.M. Humphries,

P. Hemarajata, J.D. Klausner); UCLA Health, Los Angeles

(M. Castrejon, J.D. Klausner)

DOI: https://doi.org/10.3201/eid2309.170215 infection detected by using the Cobas 4800 CT/NG Assay (Roche Molecular Systems, Pleasanton, CA, USA).

We collected data on age, sex, sex of sex partners, race/ ethnicity, HIV infection status, pregnancy, HIV preexposure prophylaxis use, gyrA gene results by anatomic site of infection, presence of other sexually transmitted infections, substance use, history of fluoroquinolone exposure in the past 2 years, and history of previous $N$. gonorrhoeae infection. Each positive $N$. gonorrhoeae result in a single patient was considered a unique infection. The date of $N$. gonorrhoeae specimen collection was considered the infection date.

Patients were considered to have no fluoroquinolone exposure only if they had medical records going back $\geq 2$ years from infection date and no documentation of having been prescribed a fluoroquinolone during that time. Patients were considered to have no history of previous $N$. gonorrhoeae infection if they had medical records going back $\geq 2$ years from current infection date and no documentation of having a positive $N$. gonorrhoeae test result at UCLA Health during those 2 years.

Descriptive statistics, prevalence ratios (PRs), and $\mathrm{p}$ values by $\chi^{2}$ test or Fisher exact test are reported. We performed analysis by using STATA software version 14.2 (StataCorp LLC, College Station, TX, USA). UCLA determined that analysis of unidentified data was exempt from ethical review.

Among 141 patients for whom $N$. gonorrhoeae genotyping was attempted, 110 (78\%) had a genotype identified; 31 (22\%) had an indeterminate genotype. Of the 110 patients who had a genotype identified, $48(44 \%)$ had a mutant genotype and $62(56 \%)$ had a wild-type genotype. Twenty (18\%) of the patients were women, 1 of whom was a transgender woman. The remaining $90(82 \%)$ patients were men, and 58 (64\%) were men who have sex with men (MSM). Forty (42\%) patients who had documentation of HIV status were infected. Sixteen women had pregnancy tests performed; $6(37 \%)$ of these women were pregnant. We obtained demographic characteristics and other factors for all patients (Table).

Among 35 patients with a gyrA mutant genotype who had medical records going back $\geq 2$ years, 9 (26\%) were exposed to fluoroquinolones during that time, compared with $6(15 \%)$ of 41 patients with wild-type gyrA genotype with medical records going back $\geq 2$ years (PR 1.4, 95\% CI $0.85-2.3 ; \mathrm{p}=0.23)$. A recent study demonstrated that treatment might be a major driver of resistance (7), and a previous study demonstrated that a history of fluoroquinolone exposure is associated with an increased prevalence of 
Table. Characteristics of 110 patients infected with Neisseria gonorrhoeae containing gyrase A mutant and wild-type genes, UCLA Health, Los Angeles, California, USA, November 1, 2015-August 31, 2016*

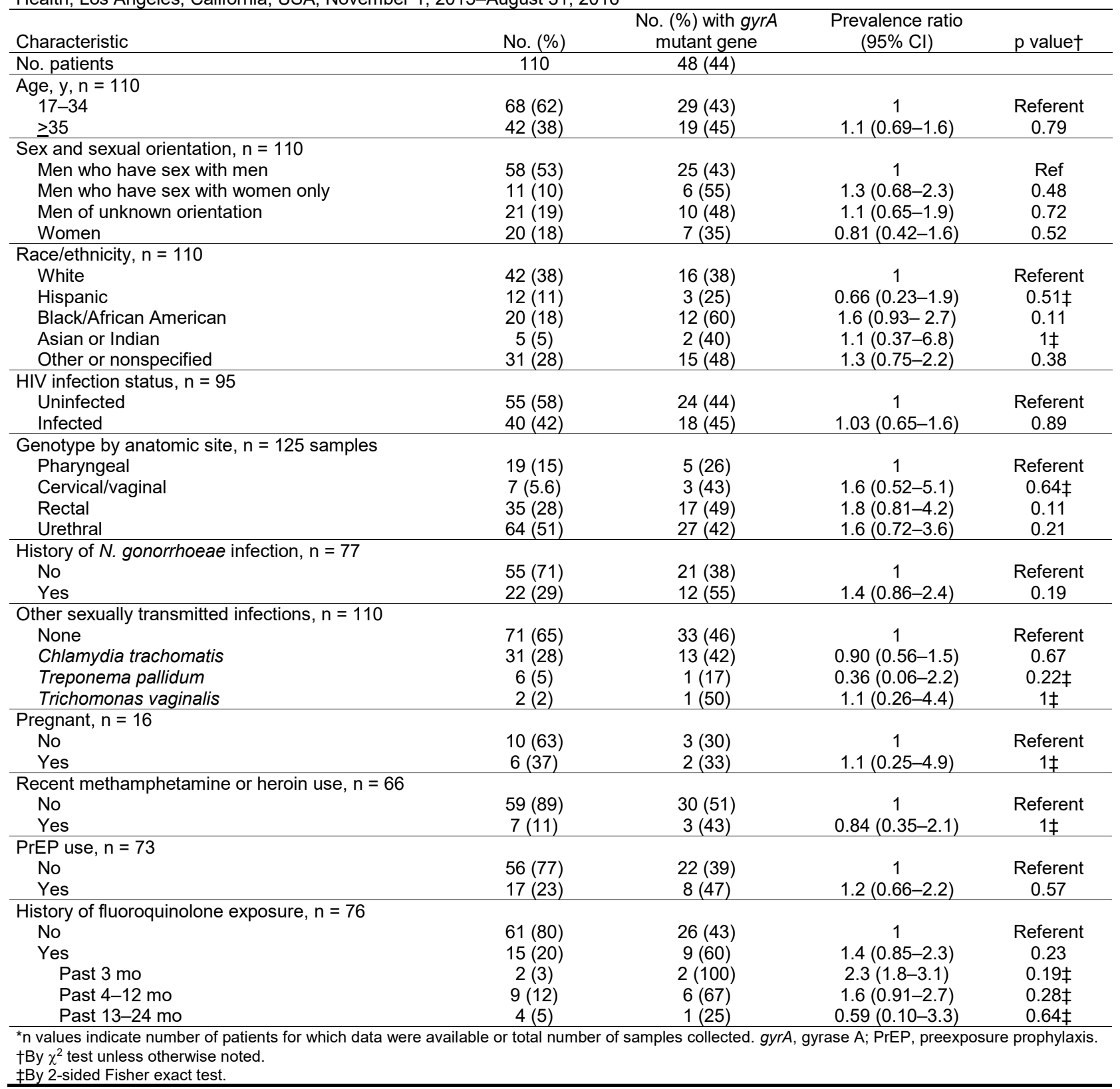

fluoroquinolone resistance (8). The lack of statistical significance between previous fluoroquinolone exposure and presence of $N$. gonorrhoeae mutant gyrA genotype in our study might be caused by small sample size.

Patients $\geq 35$ years of age were more likely to have medical records going back $\geq 2$ years than persons 17-34 years of age (PR 1.6, 95\% CI 1.2-2.0; p $<0.001)$. Men were more likely than women to have medical records going back $\geq 2$ years (PR 1.6, 95\% CI 1.0-2.5; $\mathrm{p}=0.048$ ). MSM were more likely to have medical records going back $\geq 2$ years than all other men (PR 1.4, 95\% CI 1.06-1.9; $\mathrm{p}=0.019)$. These findings indicate the possibility of selection bias regarding medication exposure in our study.

During the 10-month study period, there were 171 sitespecific $N$. gonorrhoeae infections. For these infections, none of the patients with infections at multiple anatomic sites showed discordant genotypes. One patient with repeat infections during the study showed a change in gyrA genotype from wild-type to mutant during the subsequent infection. There were no differences in distribution of genotype among anatomic sites (Figure, panel A; Table). Most infections were successfully genotyped regardless of anatomic 


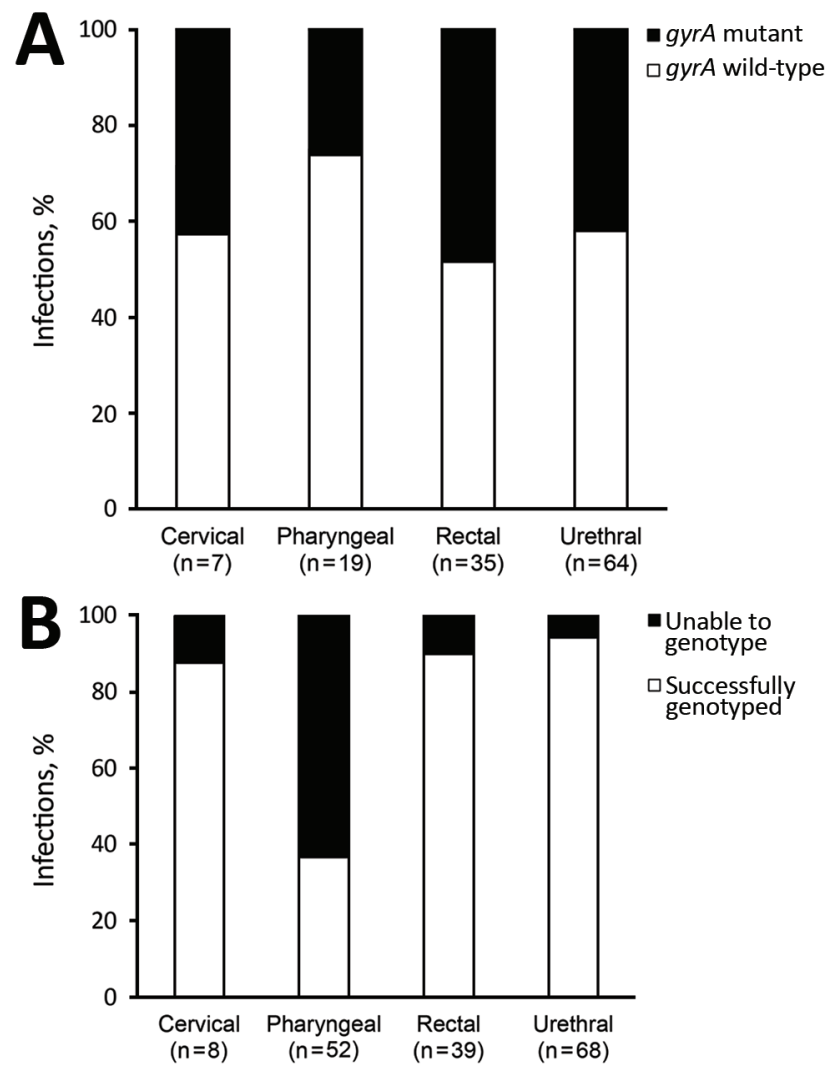

Figure. Proportion of Neisseria gonorrhoeae infections genotyped for gyrase A gene by anatomic site, UCLA Health, Los Angeles, California, USA, November 1, 2015-August 31, 2016. A) Gyrase A gene; B) gyrase A mutant and wild-type genes.

site, except for the pharyngeal site, which yielded genotypes for only $37 \%$ of infections (Figure, panel B).

Of the 42 infections that could not be genotyped, 32 were in 31 patients who had no other genotyped infections. These infections were excluded from our analyses. The remaining 10 infections were in patients who had a genotyped infection at another anatomic site: 9 of these patients were MSM and 1 was a woman. There was no major difference in distribution of sexual orientation, age, or genotype for indeterminate samples with identified genotypes for another simultaneous infection compared with those that did not have successful genotyping of another simultaneous infection.

\section{Conclusions}

Previous studies have reported a similar low sensitivity of the $N$. gonorrhoeae gyrA gene assay for pharyngeal specimens, but the cause remains uncertain $(6,9)$. It was previously believed that PCR inhibitors were present in pharyngeal reservoirs, perhaps from commensal Neisseria spp.; however, this suggestion is no longer believed to be the case $(6,9)$.
Our study had some limitations. First, patients who had medical records that go back $<2$ years might have received fluoroquinolones or had $N$. gonorrhoeae infections diagnosed at other institutions. If we were able to access that information, we would probably see a stronger association between mutant gyrA genotype and fluoroquinolone exposure and history of $N$. gonorrhoeae infection. Second, the UCLA Health system is a large, well-established institution in a large metropolitan area. Therefore, our results might not be representative of trends in $N$. gonorrhoeae resistance in other regions or healthcare systems.

In summary, we found a prevalence of $44 \%$ for the mutant gyrA genotype, which confers ciprofloxacin resistance, among patients infected with $N$. gonorrhoeae. Molecular methods to predict susceptibility testing offer a potential new way to monitor $N$. gonorrhoeae drug resistance in the United States. Replication of our work in other settings is urgently needed.

This study was supported by University of California, Los Angeles; the National Institutes of Health (grants R21AI117256 and R21AI109005); and the South American Program in HIV Prevention Research of the National Institute of Mental Health/ National Institutes of Health (grant R25MH087222).

Ms. Bhatti is a registered nurse and master's degree candidate at UCLA Fielding School of Public Health, Los Angeles.

Her research interests include sexually transmitted infections, especially in marginalized populations.

\section{References}

1. Barton J, Braxton J, Darlene D, de Voux A, Flagg E, Grier L, et al. Division of STD Prevention, Centers for Disease Control and Prevention. Sexually transmitted disease surveillance 2015. Atlanta. October 2016 [cited 2017 Jun 12]. https://www.cdc.gov/ std/stats15/std-surveillance-2015-print.pdf

2. Allan-Blitz LT, Humphries RM, Hemarajata P, Bhatti A, Pandori MW, Siedner MJ, et al. Implementation of a rapid genotypic assay to promote targeted ciprofloxacin therapy of Neisseria gonorrhoeae in a large health system. Clin Infect Dis. 2016;64:1268-70. http://dx.doi.org/10.1093/cid/ciw864

3. Allan-Blitz LT, Wang X, Klausner JD. Wild-type gyrase A genotype of Neisseria gonorrhoeae predicts in vitro susceptibility to ciprofloxacin: a systematic review of the literature and meta-analysis. Sex Transm Dis. 2017;44:261-5. http://dx.doi.org/10.1097/OLQ.0000000000000591

4. Garrett TA, Davies-Cole J, Furness B. Laboratory capacity for antimicrobial susceptibility surveillance of Neisseria gonorrhoeae, District of Columbia, 2007-2012. Sex Transm Dis. 2015;42:413-6. http://dx.doi.org/10.1097/OLQ.0000000000000304

5. Hook EW III. Generalized testing for gonococcal antibiotic susceptibility or sentinel surveillance. Sex Transm Dis. 2015;42:417-8. http://dx.doi.org/10.1097/OLQ.0000000000000321

6. Hemarajata P, Yang S, Soge OO, Humphries RM, Klausner JD. Performance and verification of a real-time PCR assay targeting the gyrA gene for prediction of ciprofloxacin resistance in Neisseria gonorrhoeae. J Clin Microbiol. 2016;54:805-8. http://dx.doi.org/10.1128/JCM.03032-15 
7. Fingerhuth SM, Bonhoeffer S, Low N, Althaus CL. Antibioticresistant Neisseria gonorrhoeae spread faster with more treatment, not more sexual partners. PLoS Pathog. 2016;12:e1005611. http://dx.doi.org/10.1371/journal.ppat.1005611

8. Klausner JD, Aplasca MR, Mesola VP, Bolan G,

Whittington WL, Holmes KK. Correlates of gonococcal infection and of antimicrobial-resistant Neisseria gonorrhoeae among female sex workers, Republic of the Philippines, 1996-1997. J Infect Dis. 1999;179:729-33. http://dx.doi.org/ $10.1086 / 314625$
9. Low N, Unemo M. Molecular tests for the detection of antimicrobial resistant Neisseria gonorrhoeae: when, where, and how to use? Curr Opin Infect Dis. 2016;29:45-51. http://dx.doi.org/10.1097/QCO.0000000000000230

Address for correspondence: Jeffrey D. Klausner, Department of Medicine, Division of Infectious Disease, University of California, Los Angeles, 10833 Le Conte Ave, Los Angeles, CA 90095, USA; email: jdklausner@mednet.ucla.edu

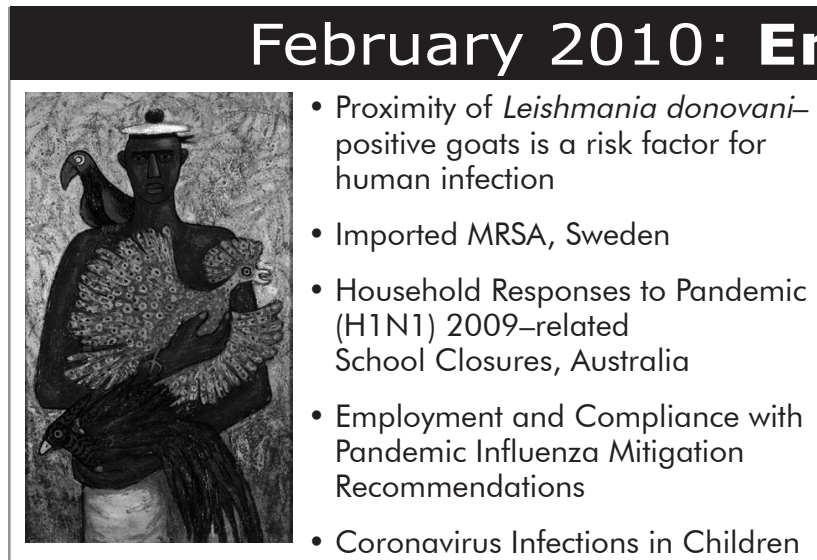

- Community-associated Clostridium difficile Infection, North Carolina

- Human Hendra Virus Encephalitis and Equine Outbreak, Australia, 2008

- Cost-effectiveness of Pharmaceutical-based Pandemic Mitigation Strategies

- Domestic Animals and Visceral Leishmaniasis, Nepal

- Influenza Vaccination Program for Children, Hawaii

- Cryptococcus gattii, British Columbia, Canada, 1999-2007

- Tropheryma whipplei in Patients with Pneumonia

- Increased Resistance in Tuberculosis Despite Treatment Adherence, South Africa

- Association Between Mycobacterium tuberculosis Strains and Phenotypes

- Cause of Epidemic among Native Americans, New England, 1616-1619

- Clonal Distribution of Invasive Pneumococci, Czech Republic, 1996-2003

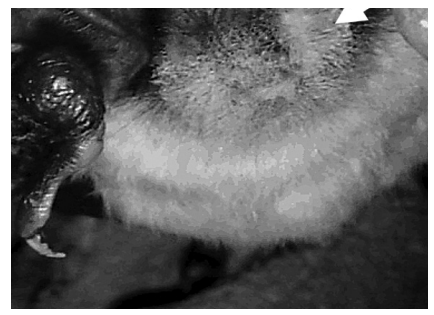

- White-Nose Syndrome Fungus in Bat, France

- Nontuberculous Mycobacteria, Taiwan, 2000-2008

\section{merging Zoonoses}

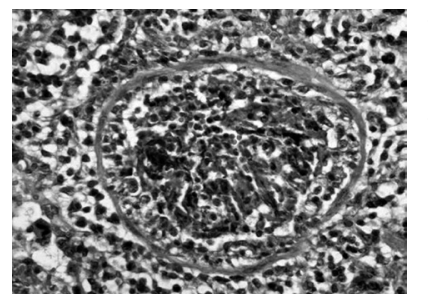

- Tularemia Outbreak, Germany

- Bordetella pertussis Clones Identified by Multilocus VariableNumber Tandem-Repeat Analysis

- Plasmodium falciparum Malaria, Southern Algeria, 2007

- Pandemic (H1N1) 2009 Outbreak on Pig Farm, Argentina

- Sin Nombre Virus Infections in Field Workers, Colorado

- Pandemic (H1N1) 2009 Cases, Buenos Aires, Argentina

- Mammalian Ancestry of Pandemic (H1N1) 2009 Virus

- Concurrent Silicosis and Mycosis at Death

- Coccidioidiomycosis among College Students, Arizona

- Novel Human Bocavirus in Children with Acute Respiratory Tract Infection

- Lymphocytic Choriomeningitis Virus Meningitis, New York, NY, 2009

- Severe Leptospirosis in Hospitalized Patients, Guadeloupe

- Enterocytozoon bieneusi

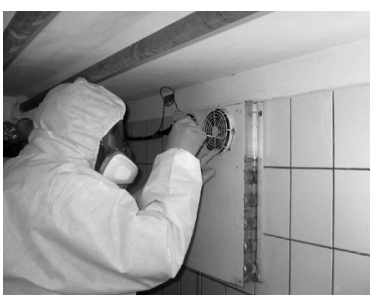
Infection, Czech Republic

- Hendra Virus Outbreak with Novel Clinical Features, Australia, 2008

- Permanent Specimens of Hosts and Vectors in Public Health and Epidemiology

- Perinatal Pandemic (H1N1) 2009 Infection, Thailand

- Bronchial Casts and Pandemic (H1N1) 2009 Virus Infection

- Methicillin-Resistant Staphylococcus aureus ST398, Italy 